\title{
Live Attenuated Influenza Vaccine
}

National Cancer Institute

\section{Source}

National Cancer Institute. Live Attenuated Influenza Vaccine. NCI Thesaurus. Code C96402.

A vaccine that contains live attenuated influenza virus (LAIV) particles. This vaccine is administered intranasally for influenza prophylaxis. Not all populations are good candidates for LAIV; the very young, elderly, asthmatics and those with health impaired may be better treated with the trivalent inactivated vaccine that is administered intramuscularly. 